
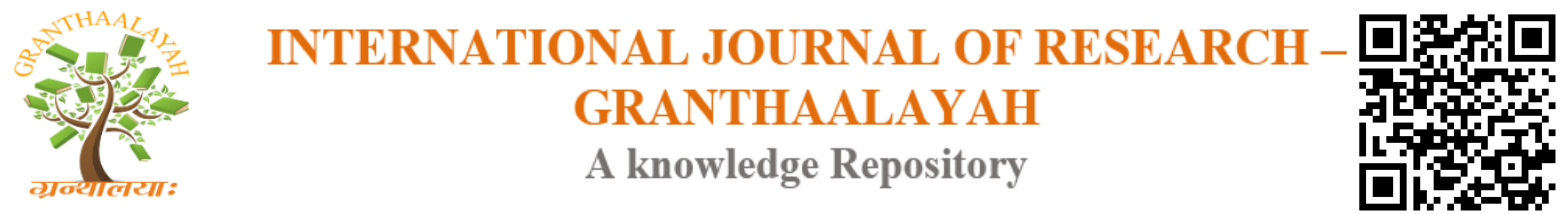

Science

\title{
RADIATION THERAPY OF HUMAN KIDNEY TISSUE BY ALPHA PARTICLES
}

\author{
Taghreed Abdull Jabbar Younis ${ }^{1}$, Firas Mahmood Hady ${ }^{2}$ \\ ${ }^{1}$ Department of Physics, College of Education for Pure Sciences -Ibn Al- Hiatham, University \\ of Baghdad, Iraq \\ ${ }^{2}$ Department of Physics, College of Science, University of Diyala, Iraq
}

\begin{abstract}
One of the most common diseases at present is the cancer that affects any member of the human body such as kidney tissue. Nuclear medicine is important in the examination and treatment, which uses a lot of particles in treatment such as Alpha particle. Alpha particles have a distinctive and important characteristic in the radiation therapy of the target part (the injured). This characteristic is due to its high ability to ionize the substance (the tissue) as it passes through, making their path short. However, there are many problems in radiation therapy; including damage to the area surrounding the tumor (the affected area) so it is very important to regulate the radiation carefully and accurately. In the present study, the study of the interaction of alpha particles with kidney tissue where the stopping power of the alpha particles was calculated using two methods: Zigler formula and SRIM software also the Range and liner energy transfer (LET) and kidney tissue thickness as well as Dose and Equivalent Dose for this particle were calculated by using Mat lab language for (0.001-1000) MeV alpha energy.
\end{abstract}

Keywords: Alpha Particles; Mass Stopping Power; SRIM Program; Zigler Formula; Range of Alpha Particle; LET; Dose; Equivalent Dose; Thickness of Kidney Tissue; Kidney Tissue.

Cite This Article: Taghreed Abdull Jabbar Younis, and Firas Mahmood Hady. (2019). "RADIATION THERAPY OF HUMAN KIDNEY TISSUE BY ALPHA PARTICLES." International Journal of Research - Granthaalayah, 7(7), 61-69. https://doi.org/10.29121/granthaalayah.v7.i7.2019.717.

\section{Introduction}

The main mechanism of loss of charged heavy particle energy is ionization or irritation. The interaction that causes the ionization or irritation processes is called the Coulomb force, which occurs between the falling particle and the electron of atom matter [1]. In order to measure the absorbed dose of ionized radiation we need to know the number of particles or photons, the amount of energy passing through the medium, the amount of energy transfer from the particle falling to the particle charged at the target, the rate of energy transfer of charged particles in the medium to the medium itself (stopping power leading to absorbed dose) [2]. 


\section{Theory}

The total stopping power of alpha particle can be obtained by using the SRIM program, which includes quick calculations that appear as tables of the stopping power and a range of particles in the material and straggling distribution of any ion in any element target [3]. The other method used in this study is Zigler's formula. In this way the energy loss process is divided into two parts: the first loss of electronic energy and the second the loss of elastic energy to the screened nuclei. In this study, the stopping power of the alpha particle was calculated using Zigler equation for the low and high energies published in the reference [3]

With regard to a compound or mixture that consists of thin layers of pure elements in the right proportion [4].

$\left(-\frac{d E}{d X}\right)_{c o m p}=\sum_{i} W_{i}\left(\frac{d E}{d X}\right)_{i} \ldots \ldots \ldots \ldots$

Where: $W_{i}=$ weight fraction,$i=$ element

The Range of the particle charged:

Range is the distance traveled by the particle in the medium or target before losing all its energy.

$R=\int_{0}^{E_{k}} \frac{d E_{k}}{(-d E / d X)} \ldots \ldots \ldots$

A plot of energy loss along the path of the particle charge is called a Bragg curve. In most of the path, the charge on the alpha particle is two electronic charges, and the amount of energy loss increases approximately as $1 / \mathrm{E}$ as predicted by Beth equation. Near the end of the path, the charge is reduced by electron capture and the curve falls off [5].

\section{Linear Energy Transfer (LET)}

Linear energy transfer (LET) is an important fundamental in radiation physics, radiation biology, and dosimetry. The mass stopping power and the transfer of linear energy are closely related to the dose and biological effectiveness of different types of radiation.

$L E T=(-d E / d X) \times \rho \ldots \ldots$

Where $\boldsymbol{\rho}=$ the density of the material or target or tissue

\section{Thickness of Target}

The thickness of the material or target penetrated by the particle charge can be calculated from the following relationship:

$T=\frac{R}{\rho} \ldots \ldots \ldots$

(4) $[6]$. 
Absorbed Dose

The absorbed dose refers to the amount of energy deposited per gram of the material [7].

$\operatorname{absorbed} \operatorname{dose}(D)=\frac{d \varepsilon}{d m} \ldots \ldots \ldots \ldots$

Where $\boldsymbol{d} \boldsymbol{\varepsilon}$ is the average energy that is transferred to the material at a small size $\mathrm{dv}$ at a point of interest in a material density over a given period of time by ionizing radiation and is the mass in dv [8].

Equivalent dose: Is the absorbed doses in the tissue or organ due to radiation [9], [10].

$H_{T}=\sum_{R} W_{R} \times D \ldots \ldots$

Where $W_{R}=$ weighting factor $=\mathbf{2 0}$ for alpha partcle [11].

Weighting factor represents a conservative judgment of envelope experimental of particle relevance to low level human exposure.

\section{Results and Discussion}

In the current study we calculated the mass stopping power of alpha particles and their Range in the human kidney tissue with energy interval (0.01-1000) MeV using Zigler equation for alpha particles and the SRIM program. It is know that the chemical compositions of human tissues are of importance in studying micro-diametric distribution in human irradiated with radiation [12], also menstruation liner energy transfer LET , and kidney thickness as well as dose , and dose equivalent for this particle were calculated by using mat lab language, the following shapes illustrate these measurements:

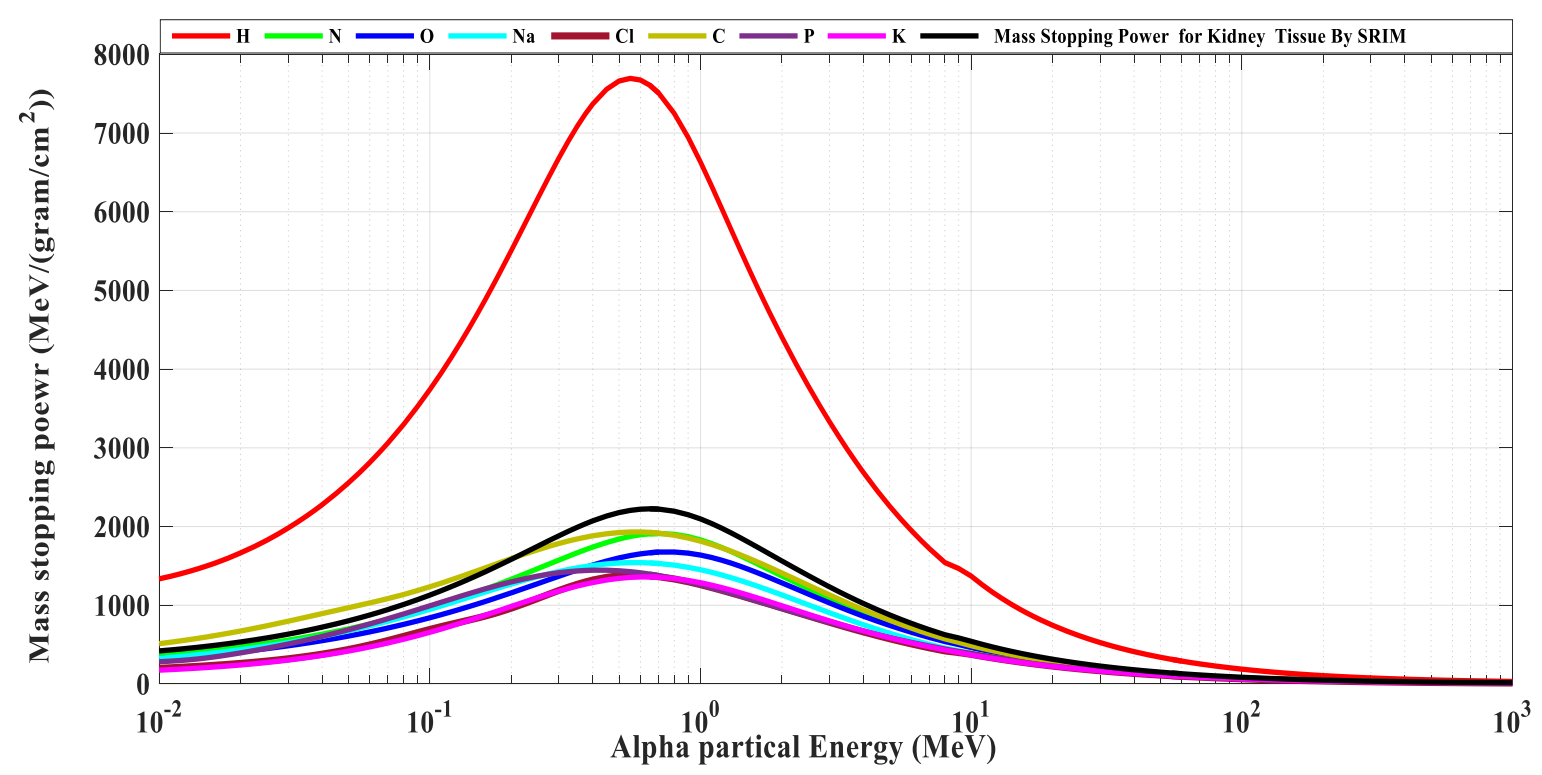

Figure 1: Mass stopping power for alpha particle in the kidney tissue by SRIM program. 


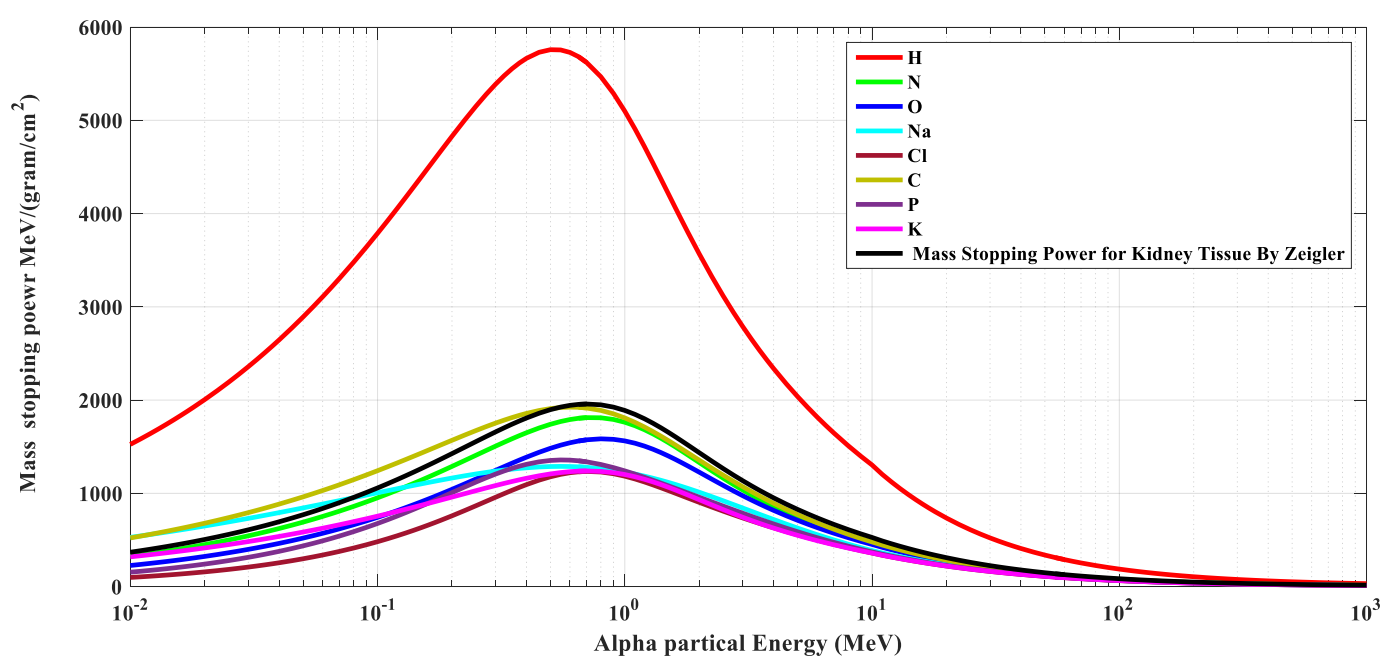

Figure 2: Mass stopping power for alpha particle in the kidney tissue by Zigler method.

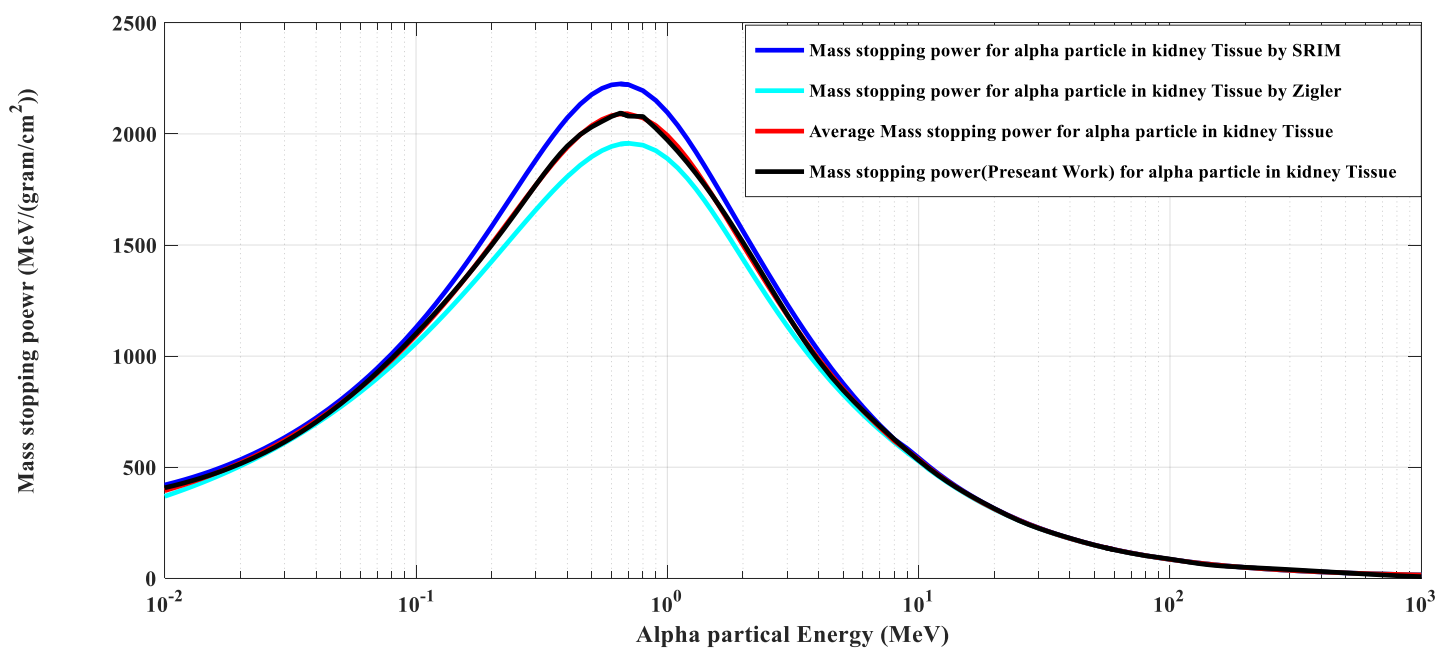

Figure 3: Mass stopping power (present work) for alpha particle in the kidney tissue.

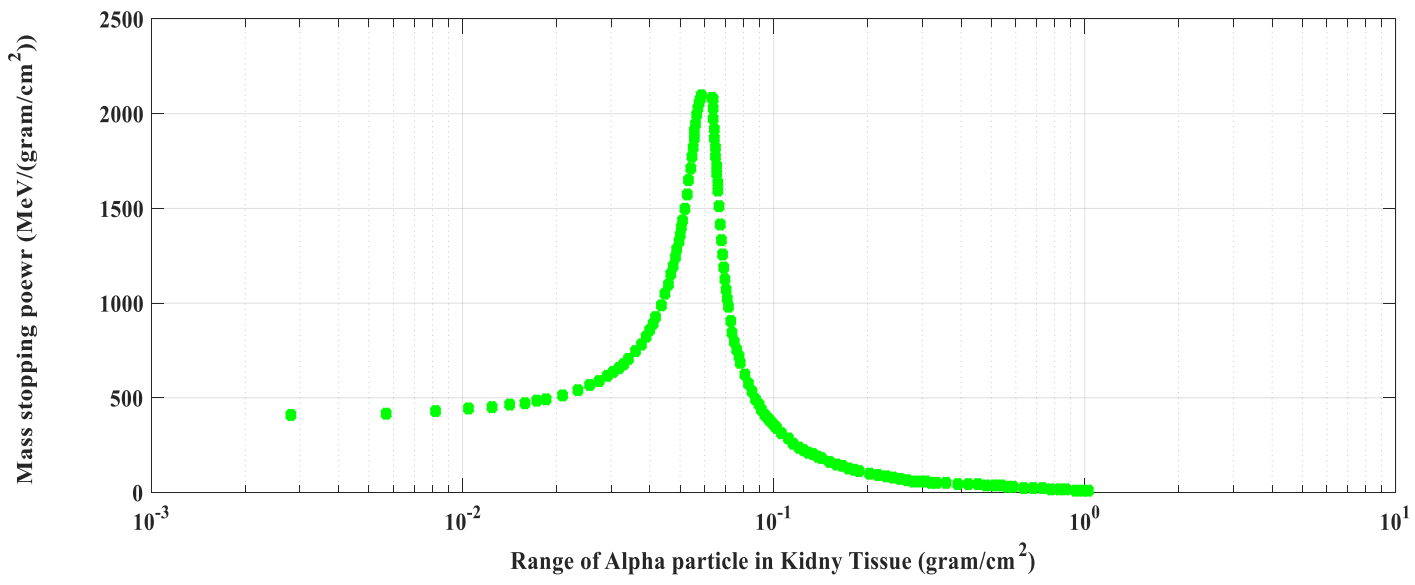

Figure 4: Range of alpha particles as a function mass stopping power (P.W) in kidney tissue . 


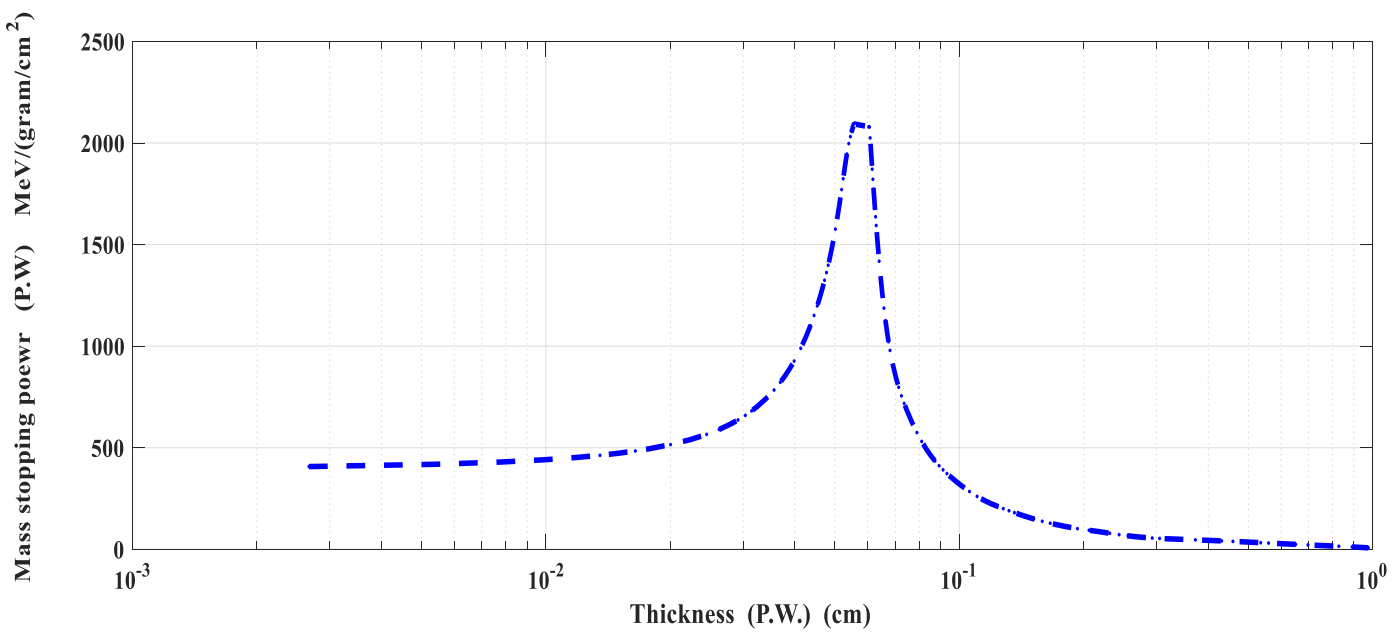

Figure 5: Thickness of target as a function mass stopping power (P.W.) in kidney tissue.

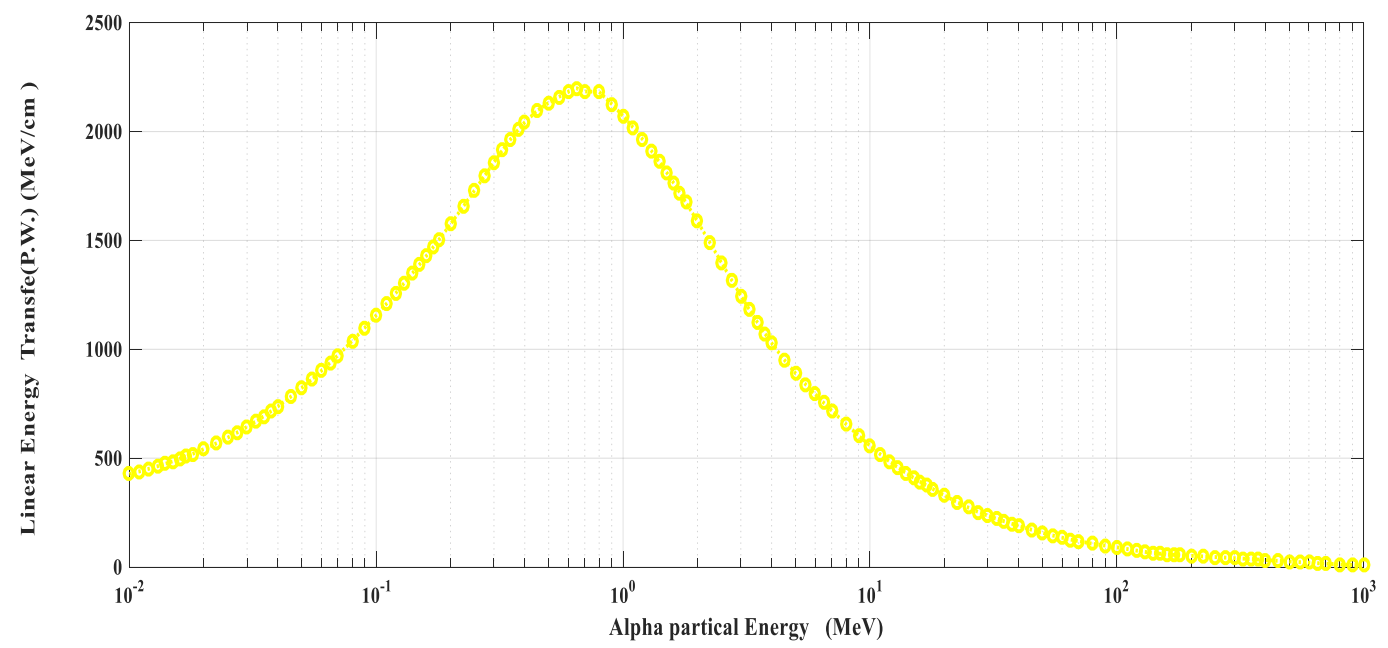

Figure 6: linear energy transfer as a function to alpha particle energy for in kidney tissue.

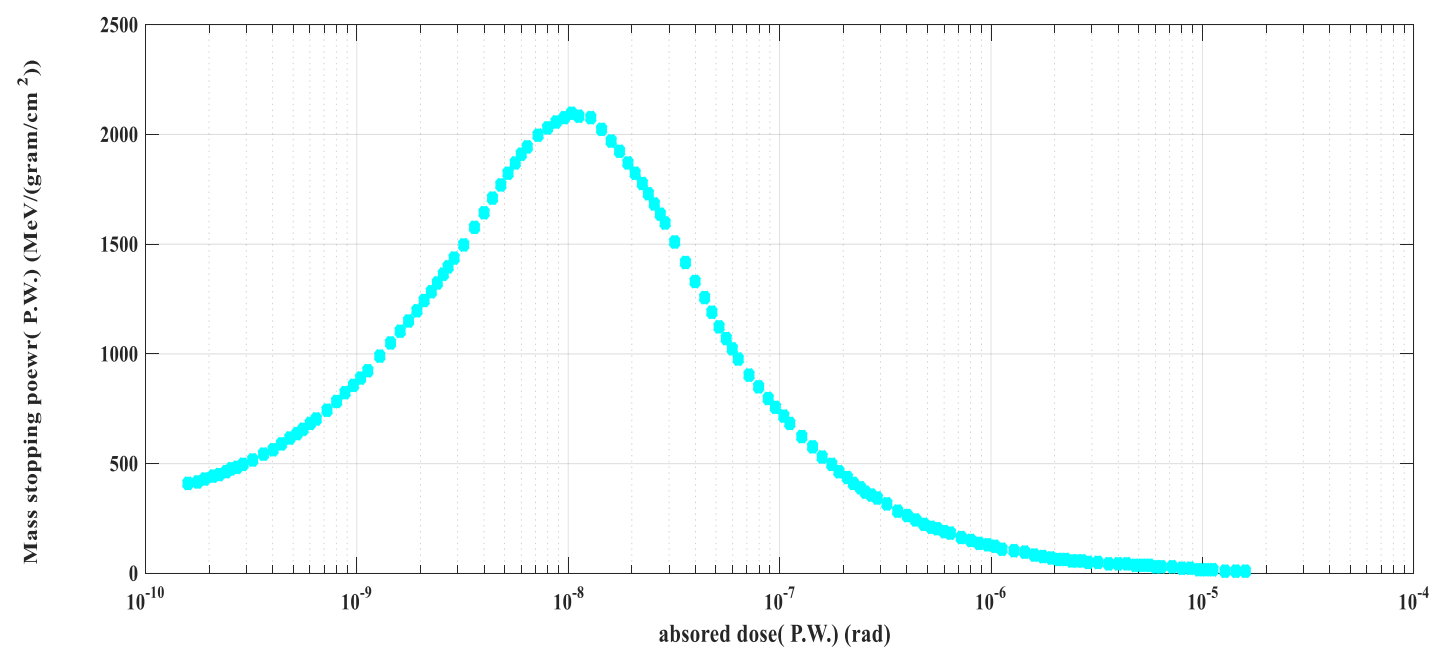

Figure 7: the maximum of absorbed doses for kidney tissue. 


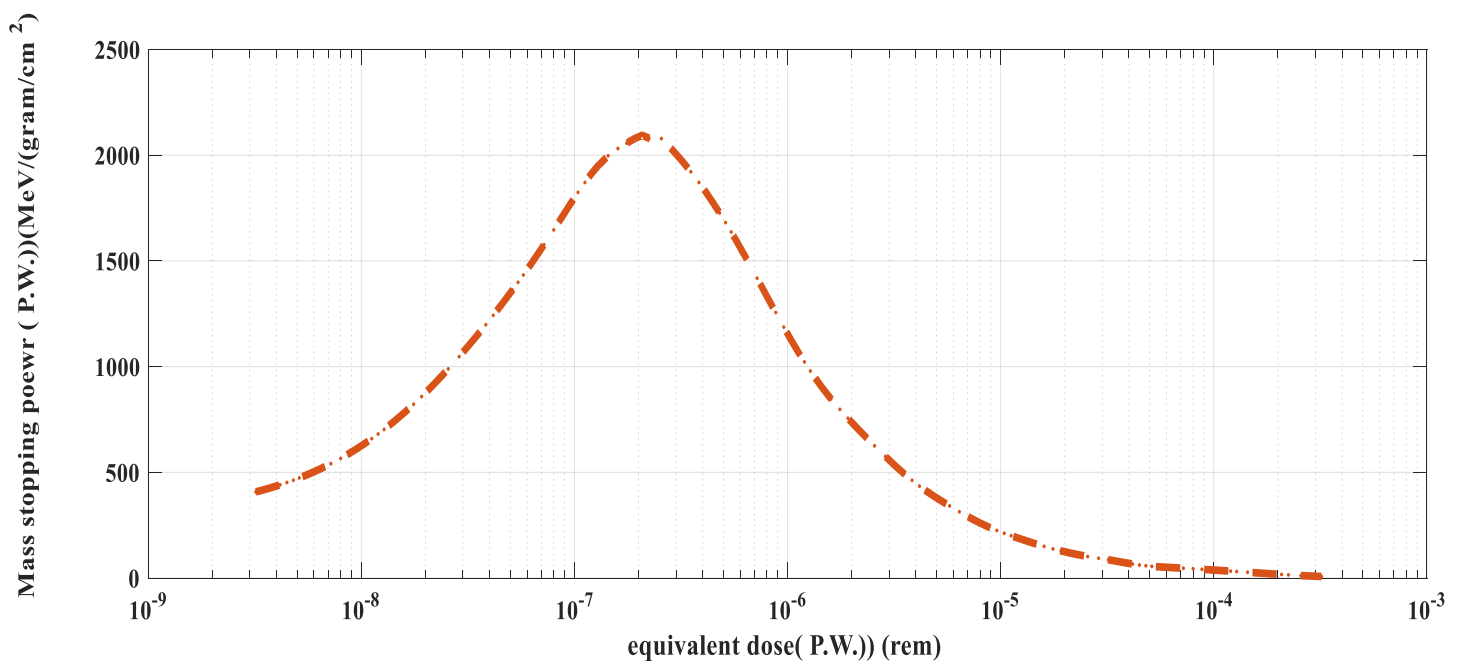

Figure 8: The Maximum of equivalent dose for kidney tissue.

1) At low energy: We find the in Ziegler's formula, SRIM program the mass stopping power increases rapidly at low energies reaches a maximum.

2) At high energy: We find the in Ziegler's formula, SRIM program the mass stopping power decreases gradually with increasing energy

3) A slow alpha particle (low energy) loses more energy by ionizing atoms than a fast alpha particle (high energy), since the slower particle spends a longer time in an atom, and thus there is a greater probability that an electronic transition will occur in the atom.

4) The maximum value of mass stopping power at the same energy is found in Hydrogen element, because Hydrogen was molecules in the traversing path of the alpha particle ions and hence the more probability of interaction and more energy lost, these results were studied by [13]. We conclude that the Hydrogen atoms are most responsible to Energy losing in the human tissues.

5) The semi-empirical formula for mass stopping power by calculation of weighted average for mass stopping power were calculate by using Matlab software, compared with two methods in kidney tissue :

1- $E<=0.75$

Linear model Poly6:

$$
\begin{aligned}
& \text { sp. }(i)=p_{1} E(i)^{6}+p_{2} E(i)^{5}+p_{3} E(i)^{4}+p_{4} E(i)^{3}+p_{5} E(i)^{2}+p_{6} E(i)+p_{7} \\
& p 1=-2.323 \times 10^{+5} \\
& p 2=5.431 \times 10^{+5} \\
& p 3=-4.991 \times 10^{+5} \\
& p 4=2.325 \times 10^{+5} \\
& p 5=-6.297 \times 10^{+4} \\
& p 6=1.255 \times 10^{+4} \\
& p 7=287.9 \\
& 1-E>0.75
\end{aligned}
$$


General model Gauss7:

$$
\begin{aligned}
a_{1} & =1924 \\
b_{1} & =-2.25 \\
c_{1} & =3.505 \\
a_{2} & =1127 \\
b_{2} & =-6.723 \\
c_{2} & =11.81 \\
a_{3} & =40.56 \\
b_{3} & =14.59 \\
c_{3} & =8.79 \\
a_{4} & =695.9 \\
b_{4} & =-58.36 \\
c_{4} & =70.11 \\
a_{5} & =-1.849 \\
b_{5} & =79.65 \\
c_{5} & =11.84 \\
a_{6} & =23.85 \\
b_{6} & =80.34 \\
c_{6} & =46.41 \\
a_{7} & =2.993 \times 10^{+15} \\
b_{7} & =-2.675 \times 10^{+4} \\
c_{7} & =4784
\end{aligned}
$$

sp. $(i)=a_{1} \exp \left(-\left(\left(x-b_{1}\right) / c_{1}\right)^{2}\right)+a_{2} \exp \left(-\left(\left(x-b_{2}\right) / c_{2}\right)^{2}\right)+a_{3} \exp (-((x$

$$
\begin{aligned}
& \left.\left.\left.-b_{3}\right) / c_{3}\right)^{2}\right)+a_{4} \exp \left(-\left(\left(x-b_{4}\right) / c_{4}\right)^{2}\right)+a_{5} \exp \left(-\left(\left(x-b_{5}\right) / c_{5}\right)^{2}\right) \\
& +a_{6} \exp \left(-\left(\left(x-b_{6}\right) / c_{6}\right)^{2}\right)+a_{7} \exp \left(-\left(\left(x-b_{7}\right) / c_{7}\right)^{2}\right)
\end{aligned}
$$

We found that the maximum value of energy the alpha particles can lose along its path in kidney tissue is $\left(2.0940 \times \mathbf{1 0}^{+3} \mathrm{MeV} . \mathrm{cm} 2 /\right.$ gram $)$, which correspond to the energy $0.65 \mathrm{MeV}$. Figure (3) illustrates the mass stopping power present work.

6) The following semi-empirical equation obtained for Range of particles in kidney tissue:

$$
1-E<=0.65 \mathrm{MeV}
$$

General model Power2:

$$
\begin{gathered}
\boldsymbol{R}(\boldsymbol{i})=\boldsymbol{a} \boldsymbol{E}(\boldsymbol{i})^{\boldsymbol{b}}+\boldsymbol{c} \\
\boldsymbol{a}=-\mathbf{0 . 0 0 6 9 8 5} \\
\boldsymbol{b}=\mathbf{- 0 . 4 8 1 6} \\
\boldsymbol{c}=\mathbf{0 . 0 6 6 9 8} \\
\mathrm{E}>0.65 \mathrm{M}
\end{gathered}
$$


Linear model Poly8:

$$
\begin{aligned}
& \mathrm{R}(\mathrm{i})=\mathrm{p}_{1} E(i)^{8}+\mathrm{p}_{2} E(i)^{7}+\mathrm{p}_{3} E(i)^{6}+\mathrm{p}_{4} E(i)^{5}+\mathrm{p}_{5} E(i) \mathrm{x}^{4}+\mathrm{p}_{6} E(i)^{3}+\mathrm{p}_{7} E(i)^{2}+ \\
& \mathrm{p}_{8} E(i)+\mathrm{p}_{9} \\
& p 1=-1.038 \times 10^{-22} \\
& p 2=4.353 \times 10^{-19} \\
& p 3=-7.435 \times 10^{-16} \\
& p 4=6.648 e \times 10^{-13} \\
& p 5=-3.337 \times 10^{-10} \\
& p 6=9.409 \times 10^{-8} \\
& p 7=-1.471 \times 10^{-5} \\
& p 8=0.002483 \\
& p 9=0.06167
\end{aligned}
$$

At low energies, the particles have a low speed, so they will stay longer in the target, so the probability of electronic transmission will increase, causing increase of loss of their energies. The slower particle spends a longer time in an atom; therefore its range will be short, but at high energies, particles have a high speed so their stay time in the target will be small therefore its range will be longer.

7) The Bragg Curve: Figures $(4,5,6,7,8)$ shows a Bragg curve. Through Bragg's curve, we could find the following:

- Near the end of the track, the charge is reduced through electron pickup, and the curve falls off.[ 14 ]

- The maximum Range of Alpha particle $\left(0.0580 \mathrm{gram} / \mathbf{c m}^{2}\right)$ in alpha energy $0.65 \mathrm{MeV}$

- The Maximum thickness of the target that the alpha particle can penetrate into kidney tissue is $(0.0556 \mathrm{~cm})$

- The maximum liner energy transfer(LET) for alpha particle that can be lost along its path in kidney tissue which corresponds to the maximum mass stopping power and maximum range and maximum penetrate thickness is $\left(2.1987 \times \mathbf{1 0}^{+3} \mathrm{MeV} / \mathrm{cm}\right)$

- The max absorbed Doses which corresponds to the Range, thickness is $\left(\mathbf{1} \times \mathbf{1 0}^{-\mathbf{8}}\right) \mathrm{rad}$

- The max Equivalent Dose $\left(\mathbf{2 0} \times \mathbf{1 0}^{-\mathbf{8}}\right) \mathrm{rem}$

Through our findings, we can provide a physician who uses an alpha particles in the treatment to help determine the amount of energy that must be used in the treatment and to determine the exact range, thickness of the target, liner energy transfer, absorbed Doses and Equivalent Dose in order to reduce the potential damage to cells adjacent to the injured Kidney tissue.

\section{References}

[1] Poskus, A., (2015), Absorption of alpha particles and electrons.

[2] Bailey, M., (2010), Ionizing radiation metrology from quantities, units and ionizing radiation fundamentals. National Physical Laboratory, Teddington, England, UK.

[3] Ziegler, J.F., (1977), IBM-Research, Helium: Stopping Powers and Ranges in all Elemental Maters, Vol. 4, Pergamon press, Yorktown Heights, New York, USA. 
[4] Leroy, C. and P.G. Rancoita, (2009), Principles of Radiation Interaction in Matter and Detection, 2nd.Edn, World Scientific Publishing Co. Pte. Ltd., Singapore, ISBN: 978-981-281-827-0, PP: 950.

[5] Knoll, G.F., (2000), Radiation Detection and Measurement. 3rd. Edn, Wiley, New York, USA, ISBN: 9780471073383 , Pages: 816.

[6] K.E.Holbert, Charged particle ionization and range, EEE460-Handout, (2012).

[7] Northwestern University, (2010), Radiation Safety Handbook office for Research Safety, Chicago, Illinois, pages: 84 .

[8] Mattsson, S. and C. Hoeschen, (2013), Radiation Protection in Nuclear Medicine, Springer, Berlin, Germany, ISBN: 978-3-642-31166-6, Pages: 161.

[9] D.Piciu, Nuclear Endocrinolo gy, 9 DOI, 10.1007/ 978-3-642-25014-9-2, Springer-Verlag Berlin Heidelberg (2012).

[10] Soren Mattson and Marcus Soderbergh, Radiation Protection in Nuclear Medicine, Springer, Verlag Berlin Heidelberg, (2013).

[11] Wrixon, A.D., (2008), New ICRP recommendations J.Radiol. Port., 28: 161-161.

[12] Kim, Y.S., (1974), Human tissues: Chemical composition and photon dosimetry data. Radiat. Res., 57: 38-45.

[13] Mahalesh D., et al. Determination of energy loss, range and stopping power of light ions using silicon surface barrier detector, International Journal of Science, Technology and Management, (2015).

[14] Glenn F.Knoll, Radiation Detection and measurement, 3rd.ed, Library of Congress Cataloging in publication (2000).

\footnotetext{
*Corresponding author.

E-mail address: firas_1962@yahoo.com
} 